\title{
Increased neutrophil-lymphocyte ratio in delirium: a pilot study
}

This article was published in the following Dove Press journal:

Clinical Interventions in Aging

14 July 2017

Number of times this article has been viewed

\author{
Angelique Egberts \\ Francesco US Mattace-Raso \\ Section of Geriatric Medicine, \\ Department of Internal Medicine, \\ Erasmus University Medical Center, \\ Rotterdam, the Netherlands
}

Aim: Delirium is a common and severe complication among older hospitalized patients. The pathophysiology is poorly understood, but it has been suggested that inflammation and oxidative stress may play a role. The aim of this pilot study was to investigate levels of the neutrophil-lymphocyte ratio (NLR) - a marker of systemic inflammation and oxidative stress - in patients with and without delirium.

Methods: This pilot study was performed within a retrospective chart review study that included acutely ill patients, 65 years and older, who were admitted to the ward of geriatrics of the Erasmus University Medical Center. All patients in whom the differential white blood cell (WBC) counts as well as the C-reactive protein (CRP) level were determined within $24 \mathrm{~h}$ after admission were included in the present study. Differences in NLR between patients with and without delirium were investigated using univariate analysis of variance, with adjustments for age, sex, comorbidities, CRP level, and total WBC count.

Results: Eighty-six patients were included. Thirteen patients were diagnosed with delirium. In adjusted models, higher mean NLR values were found in patients with, than in those without, delirium ( 9.10 vs $5.18, P=0.003$ ).

Conclusion: In this pilot study, we found increased NLR levels in patients with delirium. This finding might suggest that an inadequate response of the immune system and oxidative stress may play a role in the pathogenesis of delirium. Further studies are needed to confirm the association between NLR and delirium.

Keywords: delirium, pathology, biomarkers, leukocytes, immune system, brain

\section{Introduction}

Delirium, an acute neuropsychiatric syndrome, is a common complication among older hospitalized persons and is associated with prolonged hospital stay, loss of independence, and increased risk of cognitive decline and mortality. ${ }^{1,2}$ The underlying pathophysiology is poorly understood and the diagnosis is still primarily based on clinical observation. ${ }^{3}$ Identifying accurate biomarkers for delirium may shed light on the pathophysiology and potentially improve delirium recognition and prediction.

Both inflammation and oxidative stress may be involved in the pathophysiology of delirium. ${ }^{4,5}$ Several inflammatory markers have been investigated and were found to be associated with delirium, but time-consuming and expensive measurements make their use for research purposes and clinical practice less attractive.

The neutrophil-lymphocyte ratio (NLR), derived directly from the differential white blood cell (WBC) count, is an easily applicable marker of inflammation and oxidative stress. ${ }^{6}$ Several studies have reported an association between increased NLR and cerebrovascular disease, ${ }^{7}$ schizophrenia, ${ }^{6}$ and Alzheimer's disease ${ }^{8}$ as well as an association with increased severity and poor prognosis of various cardiovascular 
diseases $^{9-11}$ and malignancies. ${ }^{12}$ Furthermore, NLR has been found to be a more powerful predictor of cardiovascular risk and mortality in various medical conditions in comparison with traditional infection markers, such as the total WBC count, the individual WBC subtypes, and C-reactive protein (CRP). ${ }^{13-16}$ No previous study has investigated a possible association between the NLR and delirium.

We hypothesized that mean NLR levels would be elevated in patients with delirium; therefore, in this pilot study, we compared mean NLR levels of patients with and without delirium who were acutely admitted to a geriatric ward.

\section{Methods}

The present study was performed within a retrospective chart review study in which the possible association between anticholinergic drug exposure and delirium, length of hospital stay, post-discharge institutionalization, and in-hospital mortality was investigated. ${ }^{17}$ In the previous study, all acutely ill elderly aged 65 and older who were admitted to the ward of geriatrics of the Erasmus University Medical Center, Rotterdam, the Netherlands, between January 1, 2012 and December 31, 2015 were eligible for inclusion. Acutely ill patients were defined as patients with an acute disease whereby a hospital admission was required for medical treatment. Patients hospitalized for less than 3 days, admitted for elective (diagnostic) procedures, or with missing data on drug use or outcome measures were not included. ${ }^{17}$ In the present pilot study, we included all patients enrolled in the previous study in whom the differential WBC counts as well as the CRP level were determined within $24 \mathrm{~h}$ after admission. The rationale to choose this time period is that drugs, such as antibiotics, started on admission can have a significant effect on CRP and WBC counts within $48 \mathrm{~h}$. To our knowledge, it is unknown whether NLR levels are stable over time in acutely ill patients. To minimize the possible influence of drugs on the investigated markers, the first $24 \mathrm{~h}$ were chosen as the cut-off.

The study was conducted in accordance with the principles expressed in the Declaration of Helsinki. In the Netherlands, ethical approval is only required for studies in which persons are subjected to additional diagnostic procedures or treatments or are required to follow a certain behavioral strategy. No ethical approval and patient consent are required for retrospective chart review studies in which data collected during routine clinical care are extracted and analyzed anonymously.

\section{Data collection}

All data were extracted from medical records. Age, sex, place of residence before admission, and the presence of delirium during the hospital stay were documented. Severity of comorbidities on admission was calculated using the Charlson Comorbidity Index (CCI). ${ }^{18}$ The total WBC count, neutrophil count, lymphocyte count, and CRP level within $24 \mathrm{~h}$ after admission were recorded. The NLR was calculated by dividing the neutrophil count by the lymphocyte count. WBC, neutrophil, and lymphocyte counts were determined on a Sysmex XN-9000 hematology analyzer with flow cytometry (Sysmex Corporation, Kobe, Hyogo Prefecture, Japan). CRP was determined with the CRPL3 assay (immunoturbidimetric method) on a Cobas 8000 c701/702 analyzer (Roche Diagnostics, Rotkreuz, Switzerland).

\section{Definition of delirium}

Reported diagnoses of delirium were extracted from medical records. On the ward of geriatrics, the diagnosis of delirium is made by geriatricians as part of daily clinical practice, according to the criteria of the Diagnostic and Statistical Manual of Mental Disorders, 4th and 5th editions, ${ }^{19,20}$ and is based on daily psychiatric examination, medical and nursing notes, the Delirium Observation Screening scale scores, and information given by the patient's closest relative. In the previous chart review study, delirium was defined as "present on admission" if the diagnosis was made within the first 2 days of hospital stay. All other cases of delirium were defined as "incident delirium". In this pilot study, these two groups were combined.

\section{Statistical analysis}

Differences in baseline characteristics between patients with and without delirium were compared using Fisher's exact test for categorical variables, the Mann-Whitney $U$-test for nonnormally distributed continuous variables, and the Student's $t$-test for normally distributed continuous variables.

Univariate one-way analysis of variance was used to compare mean levels of NLR, CRP, WBC, neutrophils, and lymphocytes in patients with and without delirium. Biochemical parameters with a skewed distribution were logarithmically transformed using the natural log (NLR, WBC count, neutrophil count, and CRP levels). Models were adjusted for age, gender, CCI, CRP levels, and total WBC count, except when one of these variables was the dependent variable. Correlations between the aforementioned inflammatory markers were analyzed using the Spearman's correlation coefficient.

All statistical analyses were performed using the Statistical Package for the Social Sciences (SPSS), version 21.0 (IBM Corp., Armonk, NY, USA). Results were considered statistically significant at a two-sided $P$-value less than 0.05 . 
Figures were constructed using GraphPad Prism 5.01 for Windows (GraphPad Software, San Diego, CA, USA).

\section{Results}

Of the 905 patients enrolled in the chart review study, 862 had available data on the total WBC counts whereas 856 had available data on the CRP level upon admission. Moreover, in 90 patients, the differential WBC counts were determined, and 86 of them had available data on the neutrophil and lymphocyte counts as well as the CRP level within $24 \mathrm{~h}$ after admission; thus, they were included in the present pilot study.

Table 1 presents the baseline characteristics of the 86 included patients. The mean age was $80.1 \pm 6.5$ years; $37.2 \%$ were male. Thirteen patients were diagnosed with delirium, of whom eight had delirium on admission and five had incident delirium. The median number of days between admission and diagnosis of incident delirium was 6 (range: $3-13$ ). No statistically significant differences were found in age, $\mathrm{CCI}$ score, and the other demographic characteristics between patients with and without delirium.

\section{Analyses of inflammatory markers}

Mean levels and corresponding 95\% confidence intervals (CIs) of the investigated inflammatory markers in patients with and without delirium are presented in Table 2. In adjusted models, mean levels of NLR remained statistically significantly higher in patients with delirium (9.10, 95\% CI: 6.54-12.65) than in those without $(5.18,95 \% \mathrm{CI}$ : $4.53-5.93 ; P=0.003$, Figure 1). With regard to the other inflammatory markers, mean neutrophil fractions were significantly higher in patients with delirium $(78.1 \%, 95 \% \mathrm{CI}$ : $73.5-82.8)$ than in those without $(72.7 \%, 95 \%$ CI: $70.8-74.6$; $P=0.037$ ) and mean lymphocyte fractions were significantly lower in patients with delirium $(11.0 \%, 95 \%$ CI: 7.5-14.6)

Table I Baseline characteristics of the study participants

\begin{tabular}{|c|c|c|c|}
\hline Variables & $\begin{array}{l}\text { No delirium } \\
(n=73)\end{array}$ & $\begin{array}{l}\text { Delirium } \\
(n=13)\end{array}$ & $P$-value \\
\hline Male & $28(38.4)$ & $4(30.8)$ & $0.759^{\mathrm{a}}$ \\
\hline Age (years) & $79.9 \pm 6.5$ & $81.2 \pm 6.6$ & $0.517^{b}$ \\
\hline \multicolumn{4}{|c|}{ Place of residence before admission } \\
\hline $\begin{array}{l}\text { Home (with or without } \\
\text { home care) }\end{array}$ & $56(76.7)$ & $9(69.2)$ & $0.726^{\mathrm{a}}$ \\
\hline Institutional care facility & $17(23.3)$ & $4(30.8)$ & \\
\hline $\begin{array}{l}\text { First time on the ward of } \\
\text { geriatrics }\end{array}$ & $45(61.6)$ & $9(69.2)$ & $0.759^{a}$ \\
\hline Charlson Comorbidity Index & $2.0(1.0-4.0)$ & $4.0(1.5-5.5)$ & $0.110^{c}$ \\
\hline
\end{tabular}

Notes: Values are expressed as mean \pm SD for normally distributed continuous variables, median (interquartile range) for not normally distributed continuous variables and $\mathrm{n}$ (percentages) for categorical variables. 'Fisher's exact test. 'Student's t-test. 'Mann-Whitney U-test.
Table 2 Mean levels of inflammatory markers

\begin{tabular}{|c|c|c|c|}
\hline Variables & $\begin{array}{l}\text { No delirium } \\
(n=73)\end{array}$ & $\begin{array}{l}\text { Delirium } \\
(n=13)\end{array}$ & $P$-value \\
\hline $\begin{array}{l}\text { Total WBC count } \\
\left(\times 10^{9} / L\right)\end{array}$ & $9.33(8.46-10.30)$ & $7.83(6.17-9.95)$ & $0.186^{a, c}$ \\
\hline $\begin{array}{l}\text { Neutrophil count } \\
\left(\times 10^{9} / \mathrm{L}\right)\end{array}$ & $6.53(6.25-6.83)$ & $7.04(6.3 \mid-7.85)$ & $0.220^{c}$ \\
\hline $\begin{array}{l}\text { Neutrophil fraction } \\
\text { (\% of WBCs) }\end{array}$ & 72.7 (70.8-74.6) & 78.I (73.5-82.8) & 0.037 \\
\hline $\begin{array}{l}\text { Lymphocyte count } \\
\left(\times 10^{9} / \mathrm{L}\right)\end{array}$ & 1.37 (1.24-1.50) & $1.06(0.75-1.38)$ & 0.080 \\
\hline $\begin{array}{l}\text { Lymphocyte fraction } \\
\text { (\% of WBCs) }\end{array}$ & $16.3(14.8-17.7)$ & II.0 (7.5-I4.6) & 0.008 \\
\hline NLR & $5.18(4.53-5.93)$ & $9.10(6.54-12.65)$ & $0.003^{c}$ \\
\hline CRP (mg/L) & $9.7(6.2-15.3)$ & $26.3(8.8-78.3)$ & $0.099^{b, c}$ \\
\hline
\end{tabular}

Notes: Values are expressed as mean (95\% confidence intervals) and are adjusted for age, sex, Charlson Comorbity Index score, CRP level, and total WBC count unless otherwise specified. ${ }^{2}$ Not adjusted for total WBC count. ${ }^{b}$ Not adjusted for CRP level. ' 'Values are presented as the back-transformed natural log values. Abbreviations: CRP, C-reactive protein; NLR, neutrophil-lymphocyte ratio; WBC, white blood cell.

than in those without (16.3\%, 95\% CI: $14.8-17.7 ; P=0.008)$. No differences were found in total WBC count, neutrophil and lymphocyte counts, and CRP level between the groups, although the lymphocyte count was at the border of significance, being lower in patients with delirium than in those without $(P=0.080)$.

In the total group of patients, NLR showed a weak positive correlation with CRP ( $r=0.389, P<0.001$, Figure 2A), a moderate positive correlation with the total WBC count $(r=0.588, P<0.001$, Figure $2 \mathrm{~B}$ ), a strong positive correlation with the neutrophil count ( $r=0.738, P<0.001$, Figure $2 \mathrm{C}$ ), and a strong negative correlation with the lymphocyte count $(r=-0.638, P<0.001$, Figure 2D).

\section{Discussion}

In the present pilot study, we found elevated mean levels of NLR in elderly patients with delirium.

We are aware that no conclusions on causality can be drawn from this observational study; however, our results might suggest that an inadequate response of the immune system and oxidative stress might be both involved in the pathophysiology of delirium.

Activation of the immune system is a prominent feature of many conditions associated with delirium, such as infections and traumas. Several cytokines and inflammatory markers have already been detected in serum and cerebrospinal fluid during delirium. ${ }^{21-24}$ In various stressful situations, the physiological response of the immune system is characterized by an increase in neutrophils and a decrease in lymphocytes. ${ }^{25,26}$ Neutrophils play an important role in first-line defense during inflammation; once activated, they 


\section{A}
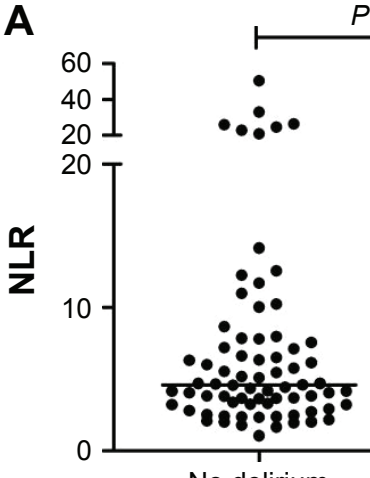

$P=0.023$
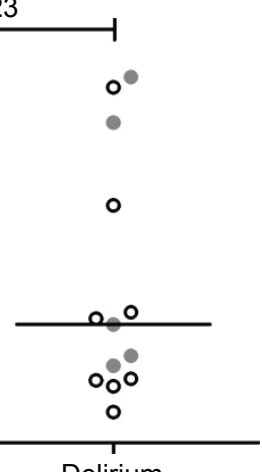

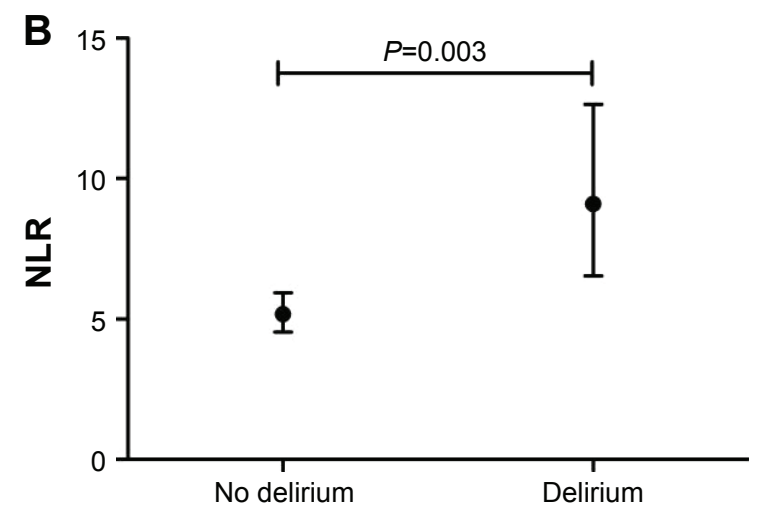

Figure I NLR in patients with and without delirium. (A) Unadjusted levels of NLR in patients with and without delirium. Lines represent medians, open dots represent patients with delirium on admission, gray dots represent patients with incident delirium. (B) Mean levels and corresponding $95 \%$ confidence intervals of NLR in patients with and without delirium. Values are back-transformed natural log values. Model is adjusted for age, gender, the Charlson Comorbidity Index score, C-reactive protein level, and white blood cell count.

Abbreviation: NLR, neutrophil-lymphocyte ratio.

release reactive oxygen species, myeloperoxidase, and proteolytic enzymes in an attempt to destroy pathogens or damaged cells. ${ }^{27,28}$

Disruption of the blood-brain barrier (BBB) and brain damage have previously been suggested as possible underlying mechanisms for delirium. ${ }^{29}$ It has been found that, at an early stage of systemic inflammation, neutrophils adhere to activated endothelial cells of the BBB, migrate across the $\mathrm{BBB}$, and release reactive oxygen species and proteases, which in turn causes destruction of the endothelial cell alignment. ${ }^{4,28,30,31}$ At the same time, several other processes increase the permeability of the BBB ${ }^{30}$ Disruption of the BBB will lead to enhanced cytokine transport into the brain. These cytokines may activate microglia, which in turn will produce a wide range of inflammatory markers and reactive oxygen species. ${ }^{32}$ Production of reactive oxygen species by neutrophils and microglia may lead to oxidative stress and may ultimately result in neuronal damage and apoptosis.
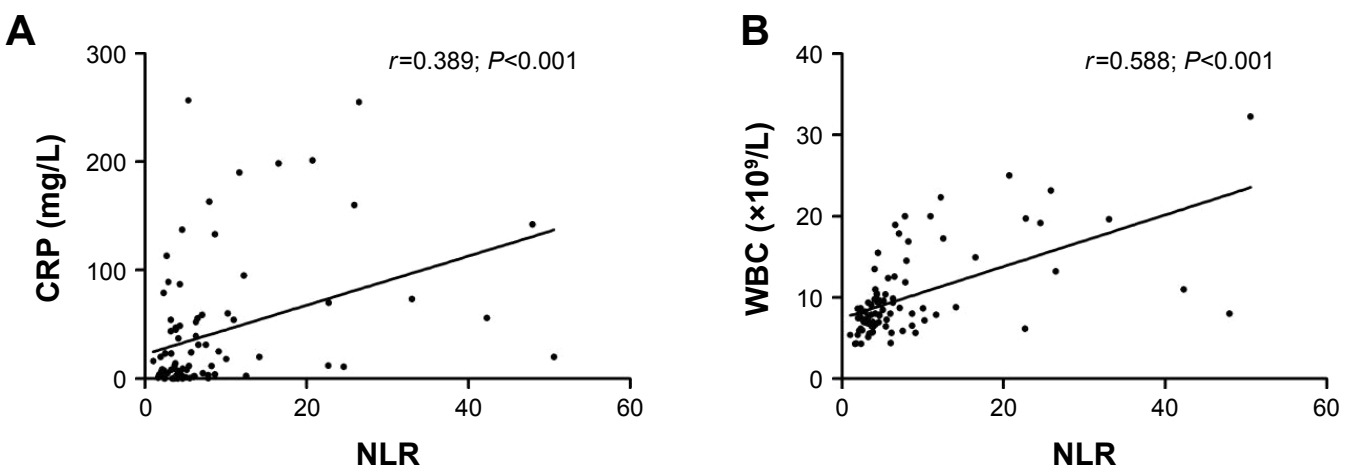

C

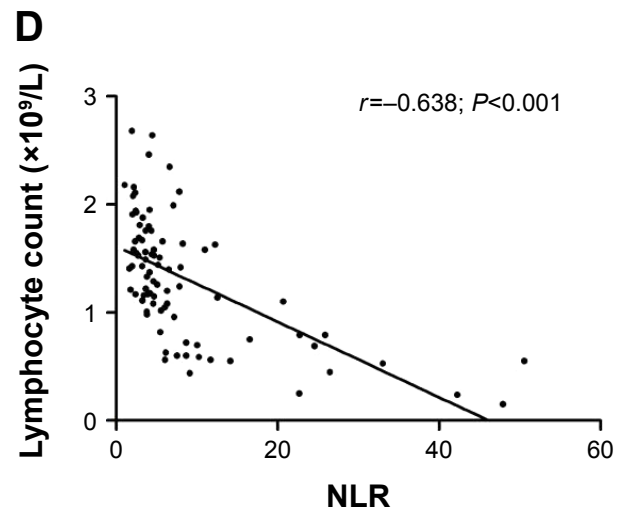

Figure 2 Correlations between the neutrophil-lymphocyte ratio (NLR) and C-reactive protein (CRP) (A), white blood cell (WBC) count (B), neutrophil count (C), and lymphocyte count (D). $r=$ Spearman's correlation coefficient. 
Lymphocytes play an important role in the regulation of an appropriate inflammatory response. Although a decrease in lymphocytes is a normal response during periods of acute stress, a chronic decrease in lymphocytes due to increased levels of catecholamine and cortisol, redistribution of lymphocytes to lymphatic tissue, and accelerated apoptosis might lead to a detrimental inflammatory state and ultimately result in poor clinical outcomes. ${ }^{25,33,34}$

NLR is the balance between neutrophils and lymphocytes and integrates two components of the immune system within one marker. In our study, as the individual neutrophil and lymphocyte counts were not (or not strongly) associated with delirium in comparison to NLR, it might be suggested that it is the balance between the two that is out of range and not particularly the two individual WBC subtypes. Therefore, an increased NLR might be suggestive for a decreased physiological reserve to respond adequately to an inflammatory insult.

In various pathological conditions, elevated NLR levels have been found to be an independent predictor of disease severity and poor prognosis. Moreover, NLR has been found to be a more powerful predictor of adverse outcomes in comparison with conventional inflammatory markers, such as the total WBC count, the individual WBC subtypes, and CRP. ${ }^{13-16}$ In the present study, we found no differences in mean levels of CRP, WBC, neutrophils, and lymphocytes between patients with and without delirium. Mean levels of NLR showed statistically significant increases in patients with delirium, even after adjustment for inflammatory markers. These findings might suggest an independent role of NLR in delirium above conventional inflammatory markers.

To the best of our knowledge, this is the first study investigating a possible association between NLR and delirium. A few studies have demonstrated that elevated NLR levels are associated with neuropsychiatric disorders such as Alzheimer's disease, ${ }^{8}$ Parkinson's disease, ${ }^{35}$ and schizophrenia ${ }^{6}$ as well as with cognitive dysfunction after carotid endarterectomy. ${ }^{36}$ Limited research has focused on the possible association of neutrophils and lymphocytes with delirium. In line with our study, Watts et al ${ }^{37}$ found no difference in neutrophil counts between patients with and without delirium who were admitted to an intensive care unit. Tanaka ${ }^{38}$ found that a decreased lymphocyte count was associated with a combined outcome of perioperative delirium and acute exacerbation of behavioral and psychological symptoms of dementia. Zuliani et a ${ }^{39}$ found that a decreased lymphocyte count was associated with subsyndromal delirium. In contrast, Inoue et a ${ }^{40}$ did not find an association between the lymphocyte count and the number of days that intensive care unit patients were free of both delirium and coma. In the present study, we found no association between the lymphocyte count and delirium, but the mean lymphocyte count seemed to be lower in patients with delirium than in those without. Of note, three of the four previous studies ${ }^{38-40}$ did not have delirium as a clear outcome, which makes it difficult to compare the results.

\section{Limitations and strengths}

This study has some limitations. First, as this was a pilot study in a relatively small group of patients, the findings need to be confirmed in a larger study. Moreover, due to the small group of patients with delirium, we were not able to perform stratified analyses for "delirium on admission" and "incident delirium". Furthermore, it might be interesting to evaluate the diagnostic and predictive power of NLR in future studies. Second, the observational design with a single measurement of NLR limits the ability to identify causal associations. Repeated NLR measurements over time are required to provide evidence for a possible role of NLR in the pathogenesis of delirium. Third, it might be speculated that other comorbidities, such as cardiovascular diseases, ${ }^{9-11}$ diabetes, ${ }^{41}$ malignancies, ${ }^{12}$ infection, ${ }^{15}$ and inflammation, ${ }^{14}$ might have influenced the mean levels of NLR; however, even after adjustment for the CCI and other inflammatory markers, estimates remained statistically significant. Fourth, only in a relatively small number of patients neutrophil and lymphocyte counts were measured on admission and this might have introduced some selection bias. Unfortunately, it is unclear why differential WBC counts were measured in these patients. The reason was probably not an increased inflammatory state, because the total WBC counts and CRP levels were lower in these patients than in the patients not included in this pilot study (data not shown).

An important strength is the use of an easily applicable marker, which combines information of several components of the immune system, as it has been suggested that the pathophysiology of delirium is multifactorial.

\section{Conclusion}

In this pilot study in older, acutely ill hospitalized patients, we found that patients with delirium had higher levels of NLR than patients without delirium. This finding might suggest that an inadequate response of the immune system and oxidative stress may play a role in the pathogenesis of delirium. Larger studies with repeated measurements of NLR over time are needed to confirm the possible role of NLR 
in the pathogenesis of delirium and to investigate whether NLR can be used as a diagnostic and predictive marker for delirium.

\section{Disclosure}

The authors report no conflicts of interest in this work.

\section{References}

1. Witlox J, Eurelings LS, de Jonghe JF, Kalisvaart KJ, Eikelenboom P, van Gool WA. Delirium in elderly patients and the risk of postdischarge mortality, institutionalization, and dementia: a meta-analysis. JAMA. 2010;304(4):443-451.

2. Siddiqi N, House AO, Holmes JD. Occurrence and outcome of delirium in medical in-patients: a systematic literature review. Age Ageing. 2006;35(4):350-364.

3. Inouye SK, Westendorp RGJ, Saczynski JS. Delirium in elderly people. Lancet. 2014;383(9920):911-922.

4. Maldonado JR. Neuropathogenesis of delirium: review of current etiologic theories and common pathways. Am J Geriatr Psychiatry. 2013;21(12):1190-1222.

5. Egberts A, Fekkes D, Wijnbeld EH, et al. Disturbed serotonergic neurotransmission and oxidative stress in elderly patients with delirium. Dement Geriatr Cogn Dis Extra. 2015;5(3):450-458.

6. Kulaksizoglu B, Kulaksizoglu S. Relationship between neutrophil/ lymphocyte ratio with oxidative stress and psychopathology in patients with schizophrenia. Neuropsychiatr Dis Treat. 2016;12:1999-2005.

7. Gökhan S, Ozhasenekler A, Mansur Durgun H, Akil E, Ustündag M, Orak M. Neutrophil lymphocyte ratios in stroke subtypes and transient ischemic attack. Eur Rev Med Pharmacol Sci. 2013;17(5):653-657.

8. Kuyumcu ME, Yesil Y, Oztürk ZA, et al. The evaluation of neutrophillymphocyte ratio in Alzheimer's disease. Dement Geriatr Cogn Disord. 2012;34(2):69-74.

9. Tamhane UU, Aneja S, Montgomery D, Rogers EK, Eagle KA, Gurm HS. Association between admission neutrophil to lymphocyte ratio and outcomes in patients with acute coronary syndrome. Am J Cardiol. 2008;102(6):653-657.

10. Wang X, Zhang G, Jiang X, Zhu H, Lu Z, Xu L. Neutrophil to lymphocyte ratio in relation to risk of all-cause mortality and cardiovascular events among patients undergoing angiography or cardiac revascularization: a meta-analysis of observational studies. Atherosclerosis. 2014;234(1):206-213.

11. Shao Q, Chen K, Rha SW, Lim HE, Li G, Liu T. Usefulness of neutrophil/lymphocyte ratio as a predictor of atrial fibrillation: a metaanalysis. Arch Med Res. 2015;46(3):199-206.

12. Templeton AJ, McNamara MG, Šeruga B, et al. Prognostic role of neutrophil-to-lymphocyte ratio in solid tumors: a systematic review and meta-analysis. J Natl Cancer Inst. 2014;106(6):dju124.

13. Horne BD, Anderson JL, John JM, et al; Intermountain Heart Collaborative Study Group. Which white blood cell subtypes predict increased cardiovascular risk? J Am Coll Cardiol. 2005;45(10):1638-1643.

14. Turak O, Özcan F, Işleyen A, et al. Usefulness of neutrophil-tolymphocyte ratio to predict in-hospital outcomes in infective endocarditis. Can J Cardiol. 2013;29(12):1672-1678.

15. de Jager CP, Wever PC, Gemen EF, et al. The neutrophil-lymphocyte count ratio in patients with community-acquired pneumonia. PLoS One. 2012;7(10):e46561.

16. Núñez J, Núñez E, Bodí V, et al. Usefulness of the neutrophil to lymphocyte ratio in predicting long-term mortality in ST segment elevation myocardial infarction. Am J Cardiol. 2008;101(6):747-752.

17. Egberts A, van der Craats ST, van Wijk MD, Alkilabe S, van den Bemt PMLA, Mattace-Raso FUS. Anticholinergic drug exposure is associated with delirium and postdischarge institutionalization in acutely ill hospitalized older patients. Pharmacol Res Perspect. 2017;5(3):e00310.
18. Charlson ME, Pompei P, Ales KL, MacKenzie CR. A new method of classifying prognostic comorbidity in longitudinal studies: development and validation. J Chronic Dis. 1987;40(5):373-383.

19. American Psychiatric Association. Diagnostic and Statistical Manual of Mental Disorders. 4th ed. Text revision. Washington, DC: American Psychiatric Association; 2000.

20. American Psychiatric Association. Diagnostic and Statistical Manual of Mental Disorders. 5th ed. Arlington, VA: American Psychiatric Association; 2013.

21. Egberts A, Wijnbeld EH, Fekkes D, et al. Neopterin: a potential biomarker for delirium in elderly patients. Dement Geriatr Cogn Disord. 2015;39(1-2):116-124.

22. Hall RJ, Watne LO, Idland AV, et al. Cerebrospinal fluid levels of neopterin are elevated in delirium after hip fracture. J Neuroinflammation. 2016;13(1):170.

23. MacLullich AM, Edelshain BT, Hall RJ, et al. Cerebrospinal fluid interleukin-8 levels are higher in people with hip fracture with perioperative delirium than in controls. J Am Geriatr Soc. 2011;59(6):1151-1153.

24. van Munster BC, Korevaar JC, Zwinderman AH, Levi M, Wiersinga WJ, De Rooij SE. Time-course of cytokines during delirium in elderly patients with hip fractures. J Am Geriatr Soc. 2008;56(9):1704-1709.

25. Zahorec R. [Ratio of neutrophil to lymphocyte counts - rapid and simple parameter of systemic inflammation and stress in critically ill]. Bratisl Lek Listy. 2001;102(1):5-14. Slovak [with English abstract].

26. Dhabhar FS, Malarkey WB, Neri E, McEwen BS. Stress-induced redistribution of immune cells - from barracks to boulevards to battlefields: a tale of three hormones - Curt Richter Award winner. Psychoneuroendocrinology. 2012;37(9):1345-1368.

27. Kolaczkowska E, Kubes P. Neutrophil recruitment and function in health and inflammation. Nat Rev Immunol. 2013;13(3):159-175.

28. Schmidt EP, Lee WL, Zemans RL, Yamashita C, Downey GP. On, around, and through: neutrophil-endothelial interactions in innate immunity. Physiology (Bethesda). 2011;26(5):334-347.

29. van Munster BC, Korevaar JC, Korse CM, Bonfrer JM, Zwinderman AH, de Rooij SE. Serum S100B in elderly patients with and without delirium. Int J Geriatr Psychiatry. 2010;25(3):234-239.

30. Varatharaj A, Galea I. The blood-brain barrier in systemic inflammation. Brain Behav Immun. 2017;60:1-12.

31. Bohatschek M, Werner A, Raivich G. Systemic LPS injection leads to granulocyte influx into normal and injured brain: effects of ICAM-1 deficiency. Exp Neurol. 2001;172(1):137-152.

32. Cerejeira J, Firmino H, Vaz-Serra A, Mukaetova-Ladinska EB. The neuroinflammatory hypothesis of delirium. Acta Neuropathol. 2010; 119(6):737-754.

33. Dhabhar FS, McEwen BS. Acute stress enhances while chronic stress suppresses cell-mediated immunity in vivo: a potential role for leukocyte trafficking. Brain Behav Immun. 1997;11(4):286-306.

34. Heffernan DS, Monaghan SF, Thakkar RK, Machan JT, Cioffi WG, Ayala A. Failure to normalize lymphopenia following trauma is associated with increased mortality, independent of the leukocytosis pattern. Crit Care. 2012;16(1):R12.

35. Akil E, Bulut A, Kaplan İ, Özdemir HH, Arslan D, Aluçlu MU. The increase of carcinoembryonic antigen (CEA), high-sensitivity C-reactive protein, and neutrophil/lymphocyte ratio in Parkinson's disease. Neurol Sci. 2015;36(3):423-428.

36. Halazun HJ, Mergeche JL, Mallon KA, Connolly ES, Heyer EJ. Neutrophil-lymphocyte ratio as a predictor of cognitive dysfunction in carotid endarterectomy patients. J Vasc Surg. 2014;59(3):768-773.

37. Watts G, Roberts BL, Parsons R. Delirium in the intensive care unit: searching for causes and sources. Crit Care Resusc. 2007;9(1):26-29.

38. Tanaka T. Factors predicting perioperative delirium and acute exacerbation of behavioral and psychological symptoms of dementia based on admission data in elderly patients with proximal femoral fracture: a retrospective study. Geriatr Gerontol Int. 2016;16(7):821-828.

39. Zuliani G, Bonetti F, Magon S, et al. Subsyndromal delirium and its determinants in elderly patients hospitalized for acute medical illness. J Gerontol A Biol Sci Med Sci. 2013;68(10):1296-1302. 
40. Inoue S, Vasilevskis EE, Pandharipande PP, et al. The impact of lymphopenia on delirium in ICU patients. PLoS One. 2015;10(5): e0126216.
41. Lou M, Luo P, Tang R, et al. Relationship between neutrophil-lymphocyte ratio and insulin resistance in newly diagnosed type 2 diabetes mellitus patients. BMC Endocr Disord. 2015;15:9.

\section{Publish your work in this journal}

Clinical Interventions in Aging is an international, peer-reviewed journal focusing on evidence-based reports on the value or lack thereof of treatments intended to prevent or delay the onset of maladaptive correlates of aging in human beings. This journal is indexed on PubMed Central, MedLine,
Dovepress

CAS, Scopus and the Elsevier Bibliographic databases. The manuscript management system is completely online and includes a very quick and fair peer-review system, which is all easy to use. Visit http://www.dovepress. $\mathrm{com} /$ testimonials.php to read real quotes from published authors. 